Int. J. Morphol.,

30(2):583-587, 2012.

\title{
Índices Antropométricos en Canoístas de Elite Jóvenes de Aguas Tranquilas
}

\author{
Anthropometric Indexes in Young Elite Male Sprint Canoeists \\ "Fernando Alacid; ***José María Muyor; "***José Ramón Alvero-Cruz; \\ ${ }^{* * * * *}$ Manuel Isorna \& ${ }^{* * * * *}$ Pedro Ángel López-Miñarro
}

ALACID, F.; MUYOR, J. M.; ALVERO-CRUZ, J. R.; ISORNA, M. \& LÓPEZ-MIÑARRO, P. A. Índices antropométricos en canoístas de elite jóvenes de aguas tranquilas. Int. J. Morphol., 30(2):583-587, 2012.

RESUMEN: El objetivo de este estudio fue describir las características de los índices antropométricos en canoístas de categoría cadete. A un total de 33 canoístas se les valoró peso, talla, envergadura, talla sentado, perímetros de la cintura y cadera, longitudes del brazo y antebrazo, así como diámetros biacromial y biileocrestal. Se calculó el índice de masa corporal (IMC), ratio cintura-cadera, envergadura relativa e índices córmico, de Manouvrier, acromio-iliaco y braquial. La mayor parte de los canoístas de categoría cadete obtuvieron valores propios de normopeso respecto al IMC, valores bajos de ratio cintura-cadera y una envergadura mayor que la altura. Existe una mayor variedad respecto al resto de índices estudiados, dándose un mayor número de casos de tronco medio para el índice córmico, extremidades inferiores largas para el índice de Manuovrier, tronco trapezoidal e intermedio para el índice acromio-iliaco y antebrazo medio y corto para el índice braquial.

PALABRAS CLAVE: Antropometría; Índices corporales; piragüismo/canotaje.

\section{INTRODUCCIÓN}

Las características morfológicas de palistas jóvenes han sido descritas en diferentes trabajos de investigación. Algunos se han centrado en la descripción de las características de raquis en el plano sagital y la extensibilidad isquiosural como factores que determinan la postura durante las acciones de paleo (López-Miñarro \& Alacid, 2010; López-Miñarro et al., 2010a, b, 2011; Muyor et al., 2011). Sin embargo, otras investigaciones han tratado aspectos relacionados con la antropometría y composición corporal de los palistas jóvenes. Arlettaz et al. (2004) compararon las masa magra y ósea mediante DXA entre kayakistas y sedentarios de 15 años de edad, encontrando una mayor masa libre de grasa y componente óseo en los kayakistas, especialmente en las partes específicas implicadas en la acción de paleo. Otros trabajos compararon las características antropométricas entre kayakistas y canoístas de categoría infantil, encontraron diferencias en la talla, el peso y los pequeños diámetros del húmero, fémur y muñeca
(Carrasco et al., 2005). Sin embargo, otros han encontrado cierta similitud entre ambas disciplinas en lo que se refiere a índices antropométricos (Alacid et al., 2011a). En esta misma categoría, pero buscando diferencias entre las edades de 13 y 14 años, Alacid et al. (2011b) las encontraron en categoría masculina en la talla, peso, talla sentado, envergadura y un buen número de perímetros, longitudes y diámetros de la extremidad superior y el tronco, siendo casi inexistentes estas diferencias en las mujeres. Sin embargo, son bastante escasos los trabajos que aborden de forma específica las características antropométricas de la disciplina de canoa canadiense en palistas jóvenes (Alacid et al., 2011c).

Aunque las disciplinas de canoa y kayak de aguas tranquilas son propias del piragüismo o canotaje, las diferencias entre ambas, en cuanto a técnica de paleo se refiere, son importantes. En kayak, el palista se encuentra senta-

\footnotetext{
Departamento de Actividad Física y Deporte. Facultad de Ciencias del Deporte. Universidad de Murcia. España.

** Área de Expresión Corporal. Facultad de Ciencias de la Educación. Universidad de Almería. España.

*** Escuela de Medicina de la Educación Física y el Deporte. Depto. de Fisiología Humana y Educación Física y Deportiva. Universidad de Málaga. España.

***** Facultad de Educación y el Deporte. Universidad de Vigo. España.

****** Departamento de Expresión Plástica, Musical y Dinámica. Facultad de Educación. Universidad de Murcia. España.

Este estudio ha sido financiado con una ayuda de la Fundación Séneca-Agencia de Ciencia y Tecnología de la Región de Murcia (II PCTRM 20072010) con $n^{\circ}$ 11951/PI/09 (Evolución de las curvaturas sagitales del raquis, extensibilidad isquiosural, dolor lumbar y características antropométricas de piragüistas de élite).
} 
do y transmite la fuerza a la embarcación a través del reposapiés y el asiento, mientras que en canoa, el palista se encuentra arrodillado sobre una sola rodilla. Además, los kayakistas palean de forma alternativa con una pala de doble hoja, manteniendo la dirección del kayak mediante un timón que es manejado con los pies, mientras que los canoístas palean por un solo lado de la embarcación con una pala simple (de una hoja) y maneja la dirección con diversas maniobras realizadas con la misma.

Los índices antropométricos o corporales son la relación entre dos medidas corporales, expresando el porcentaje de la menor sobre la mayor. Pacheco (1993) considera muy interesante el análisis de los mismos en cineantropometría, puesto que algunos presentan valores distintos en deportistas de disciplinas diferentes o edades, pudiendo ser responsables, en parte, de la aptitud hacia las diferentes pruebas atléticas, por lo que podrían ser un aspecto a tener en cuenta en la orientación y detección de talentos en diversas modalidades deportivas.

Dado que los índices antropométricos han sido muy poco tratados en las publicaciones relacionadas con el piragüismo y existe un reducido número de trabajos realizados sobre la disciplina de canoa canadiense, el objetivo de este estudio fue describir las características de proporcionalidad aportadas por los índices antropométricos en canoístas de categoría cadete.

\section{MATERIAL Y MÉTODO}

Un total de 33 hombres canoístas de categoría cadete (media \pm desviación típica; edad: 15,6 \pm 0,6 años; talla: $172,9 \pm 5,3$; peso: $68,6 \pm 7,1)$ participaron en este estudio. Todos ellos fueron seleccionados por encontrarse entre los mejores de su categoría, para su asistencia a las Concentraciones Nacionales de Cadetes en los años 2009 y 2010, realizadas dentro del Programa Nacional de Tecnificación de Cadetes del Consejo Superior de Deportes y la Real Federación Española de Piragüismo.

Los padres y los deportistas fueron informados de los objetivos y métodos del estudio y se obtuvo un consentimiento informado de sus tutores.

Las medidas utilizadas para la determinación de los índices antropométricos fueron el peso, la talla, la envergadura, la talla sentado, los perímetros de la cintura y la cadera, las longitudes del brazo y el antebrazo y los diámetros biacromial y biileocrestal. Todas ellas se tomaron siguiendo las indicaciones de la ISAK (International Society for the Advancement of Kinanthropometry) (Marfell-Jones et al., 2006), por un antropometrista acreditado de nivel II. Las medidas se tomaron dos o tres veces, si la diferencia entre las dos primeras era superior al 5\% en pliegues y al $1 \%$ en el resto de medidas, tomando la media o la mediana, respectivamente, para realizar los análisis posteriores.

Para la determinación del peso se utilizó una báscula SECA 862 (SECA, Alemania) de 100 g de precisión; para la envergadura y los perímetros una cinta métrica inextensible milimetrada Lufkin W606PM (Lufkin, EE.UU.); y para la talla, talla sentado, longitudes y diámetros, un antropómetro Siber-Hegner GPM (Suiza). Ambos instrumentos con una precisión de $0,1 \mathrm{~cm}$.

A partir de los datos obtenidos se valoraron 7 índices, dos de ellos muy usados en la valoración del estado nutricional, dos sobre las proporciones de la talla sentado y la estatura, uno sobre las del tronco, otro sobre la relación entre la talla y la envergadura y un último que analiza la relación entre el antebrazo y el brazo, según lo expresado a continuación:

- Índice de Quetelet o índice de masa corporal: se obtiene tras dividir el peso $(\mathrm{kg})$ entre el cuadrado de la talla $(\mathrm{m})$.

- Ratio cintura - cadera: relaciona el perímetro de la cintura con el de la cadera. En el ámbito clínico es considerado como uno de los mejores indicadores del riesgo cardiovascular.

. Envergadura relativa: se divide la envergadura entre la talla, multiplicando el resultado por 100.

- Índice Córmico: se divide la talla sentado entre la talla, multiplicando el resultado por 100 .

- Índice de Manouvrier: se resta la talla sentado a la talla, dividiendo el resultado entre la talla sentado y multiplicando por 100 el resultado final.

- Índice acromio-iliaco: se divide el diámetro biileocrestal entre el biacromial, multiplicando el resultado por 100.

- Índice braquial: se divide la longitud del antebrazo entre la del brazo, multiplicando el resultado por 100.

La interpretación de los dos primeros índices se realizó siguiendo las indicaciones de la Organización Mundial de la Salud (WHO, 2000), mientras que para el resto se utilizaron las propuestas por Pacheco (Tabla I). Se contabilizó el número de casos para cada uno de los morfotipos encontrados 
Tabla I. Interpretación de los índices corporales en hombres, segun Pacheco.

\begin{tabular}{|c|c|c|}
\hline Índice & Int erpretación & Resultado \\
\hline \multirow{5}{*}{$\begin{array}{l}\text { Índice de Masa } \\
\text { Corporal }\end{array}$} & Bajo peso & $<18,5$ \\
\hline & Normo peso & $18,5-24,9$ \\
\hline & Sobrepeso I & $25,0-27,4$ \\
\hline & Sobrepeso II & $27,5-29,9$ \\
\hline & Obesidad & $\geq 30,0$ \\
\hline \multirow{3}{*}{$\begin{array}{l}\text { Ratio Cintura - Cadera } \\
\text { (Riesgo cardiovascular) }\end{array}$} & Bajo & $<0,9$ \\
\hline & Moderado & $0,9-1,0$ \\
\hline & Alto & $>1,0$ \\
\hline \multirow{2}{*}{ Envergadura Relativa } & Envergadura > Talla & $<100$ \\
\hline & Envergadura $<$ Talla & $>100$ \\
\hline \multirow{3}{*}{ Índice Córmico } & Tronco corto & $\leq 51,0$ \\
\hline & Tronco medio & $51,1-53,0$ \\
\hline & Tronco largo & $\geq 53,1$ \\
\hline \multirow{3}{*}{$\begin{array}{l}\text { Índice de Manouvrier } \\
\text { (Miembros inferiores) }\end{array}$} & Cortos & $\leq 84,9$ \\
\hline & Medianos & $85,0-89,9$ \\
\hline & Largos & $\geq 90,0$ \\
\hline \multirow{3}{*}{ Índice Acromi o-iliaco } & Tronco trapezoidal & $\leq 69,9$ \\
\hline & Tronco intermedio & $70,0-74,9$ \\
\hline & Tronco rectangular & $\geq 75,0$ \\
\hline \multirow{3}{*}{ Índice B raquial } & Antebrazo corto & $\leq 77,9$ \\
\hline & Antebrazo medio & $78,0-82,9$ \\
\hline & Antebrazo largo & $\geq 83,0$ \\
\hline
\end{tabular}

\section{RESULTADOS}

Se valoraron siete índices corporales resultantes de la relación entre diversas variables obtenidas de forma directa. En la Tabla II se muestran los valores medios \pm desviación típica de los índices calculados y el número de casos según las interpretaciones de cada uno de ellos.

En lo que se refiere al índice de masa corporal, la mayor parte de los palistas obtuvieron valores correspondientes al normopeso, existiendo únicamente dos casos que fueron categorizados como sobrepeso. Los valores de la ratio cintura-cadera de la totalidad de los canoístas los situó en la categoría de riesgo cardiovascular bajo. En todos los casos excepto tres, la envergadura fue superior a la talla.

El morfotipo de tronco medio fue el más frecuente entre los canoístas cadetes con 23 casos, seguido del tronco largo con 9 y corto con un único caso.
Tabla II. Índices corporales en canoístas de categoría cadete.

\begin{tabular}{|c|c|c|c|}
\hline Índice & $\begin{array}{l}\text { Valor medio } \\
\quad \pm \mathrm{dt}\end{array}$ & $\begin{array}{l}\text { Número } \\
\text { de casos }\end{array}$ & Interpretación \\
\hline \multirow{5}{*}{ Índice de masa corporal } & \multirow{5}{*}{$22,93 \pm 1,73$} & - & Bajo peso \\
\hline & & 31 & Normo peso \\
\hline & & 2 & Sobrepeso I \\
\hline & & - & Sobrepeso II \\
\hline & & - & Obesidad \\
\hline \multirow{3}{*}{$\begin{array}{l}\text { Ratio cintura - cadera } \\
\text { (Riesgo cardiovascul ar) }\end{array}$} & \multirow{3}{*}{$0,81 \pm 0,03$} & 33 & Bajo \\
\hline & & - & Moderado \\
\hline & & - & Alto \\
\hline \multirow{2}{*}{ Envergadura relativa } & \multirow{2}{*}{$102,04 \pm 1,77$} & 30 & Envergadura $>$ Talla \\
\hline & & 3 & Envergadura $<$ Talla \\
\hline \multirow{3}{*}{ Índice córmico } & \multirow{3}{*}{$52,50 \pm 1,13$} & 1 & Tronco corto \\
\hline & & 23 & Tronco medio \\
\hline & & 9 & Tronco largo \\
\hline \multirow{3}{*}{$\begin{array}{l}\text { Índice de Manouvrier } \\
\text { (Miembros inferiores) }\end{array}$} & \multirow{3}{*}{$90,56 \pm 4,04$} & 5 & Cortas \\
\hline & & 4 & Medi anas \\
\hline & & 24 & Largas \\
\hline \multirow{3}{*}{ Índice acromi o-iliaco } & \multirow{3}{*}{$70,72 \pm 3,53$} & 15 & Tronco trapezoidal \\
\hline & & 13 & Tronco intermedio \\
\hline & & 5 & Tronco rectangular \\
\hline \multirow{3}{*}{ Índice braquial } & \multirow{3}{*}{$79,20 \pm 3,06$} & 12 & Antebrazo corto \\
\hline & & 17 & Antebrazo medio \\
\hline & & 4 & Antebrazo largo \\
\hline
\end{tabular}

Dt: desviación típica.

Para el índice de Manouvrier, la mayoría de los casos estudiados poseyeron extremidades inferiores largas (24), encontrando 4 y 5 canoístas con longitudes medianas y cortas, respectivamente para este índice. El tronco trapezoidal e intermedio fueron los más habituales en la interpretación del índice acromio-iliaco con 15 y 13 casos, respectivamente, siendo sólo 5 los palistas que presentaron un tronco rectangular. Respecto al índice braquial, la mayor parte de los casos presentaron antebrazos medios y cortos, con un solo caso de antebrazo largo.

\section{DISCUSIÓN}

El IMC de canoístas cadetes correspondió en la mayoría de los casos al normopeso y sólo dos casos aislados con sobrepeso. En los palistas de elite, los valores de este índice suelen oscilar de 23 a $24 \mathrm{~kg} \cdot \mathrm{m}^{-2}$, aunque existen trabajos en los que se sobrepasan ligeramente los $25 \mathrm{~kg} \cdot \mathrm{m}^{-2}$ (Ackland et al., 2003; Aitken \& Jenkins, 
1998; Lutoslawska et al., 1994; van Someren \& Palmer, 2003; Wisniewska et al., 1985), cifra límite a partir de la que se identifica un teórico sobrepeso (Salas-Salvado et al., 2007; WHO, 2000). En canoístas de categoría inferior (de 13 y 14 años), aunque predominaron los valores de normopeso, es destacable la presencia de un $15 \%$ de palistas con valores propios del bajo peso (Alacid et al., 2011a).

Los resultados obtenidos en la ratio cintura-cadera, sitúan a nuestros palistas en una categoría de riesgo cardiovascular bajo, al obtener valores inferiores a 0,9 , coincidiendo con los palistas que participaron en los JJ.OO. de Sydney (2000) en las especialidades de aguas tranquilas (Ackland et al.) y aguas bravas (Ridge et al., 2007) así como con los canoístas de categoría infantil (Alacid et al., 2011a). La envergadura fue mayor que la talla en el mayor número de casos. Estos resultados coincidieron con los de hombres kayakistas de elite, con valores en torno al 103,5\% (Ackland et al.; Aitken \& Jenkins), en la categoría infantil (Alacid et al., 2011a; Cuesta et al., 1991) y en la especialidad de aguas bravas (Ridge et al.).

Los valores correspondientes a la valoración como tronco medio en el índice córmico fueron los más frecuentes, seguida del tronco largo. Tras obtener esta variable, en la mayor parte de los trabajos consultados (Ackland et al.; Aitken \& Jenkins; Fry \& Morton, 1991; van Someren \& Palmer) se identifican a los palistas de elite con el morfotipo de tronco medio, y con el tronco largo en hombres kayakistas de aguas tranquilas (Cermak et al., 1975) y en hombres canoístas de outrigger (Humphries et al., 2000). En canoístas infantiles de 13 años, sin embargo, prevalece el tronco corto sobre el resto de morfotipos, pasando a predominar el tronco medio a los 14 años (Alacid et al., 2011a). Esta evolución del tronco corto a medio o largo de los 13 a los 15 años, podría ser identificada con la evolución de la talla y la talla sentado a lo largo del proceso de crecimiento. De hecho, la relación de estas medidas antropométricas fue utilizada por Mirwald et al. (2002) para determinar el tiempo que falta o que ha pasado para llegar a la edad de máxima velocidad de crecimiento en altura.

La interpretación de miembros inferiores largos fue la obtenida en un mayor número de casos en el índice de Manouvrier, coincidiendo con lo encontrado en un alto número de estudios en palistas de alto nivel (Ackland et al.; Aitken \& Jenkins; Fry \& Morton; van Someren \& Palmer), y de categoría infantil (Alacid et al., 2011a).

Los resultados encontrados respecto al índice acromioiliaco muestran una prevalencia del tronco trapezoidal en estos canoístas, coincidiendo con los resultados encontrados en canoístas de elite (Misigoj-Durakovic \& Heimer, 1992).
Sin embargo en palistas de categoría infantil no se encontraron casos de este morfotipo, predominando mayoritariamente el morfotipo de tronco rectangular (Alacid et al., 2011a), hecho que podría identificarse como resultado del proceso de desarrollo físico y de entrenamiento en la especialidad.

Respecto al índice braquial, los morfotipos predominantes fueron el de antebrazo medio y corto, lo que coincide con lo indicado por Norton \& Olds (1996) donde afirman que el índice braquial de los palistas $(79,0 \%)$ es superior al de otros deportistas $(74,8 \%)$, ya que este hecho supone una ventaja mecánica en el paleo. En este sentido, los resultados del índice braquial en hombres kayakistas (76-77\%; antebrazo corto) de elite (Ackland et al.; Aitken \& Jenkins), fueron ligeramente inferiores a los de nuestros palistas (79,2\%; antebrazo medio), por lo que probablemente sería de interés replantearse los valores que determinan cada uno de los morfotipos relacionados con la longitud del antebrazo respecto al brazo.

Como conclusión de este trabajo, los hombres canoístas de categoría cadete poseen unos índices corporales bastante homogéneos respecto al IMC, la ratio cintura-cadera y la envergadura relativa, predominando el normo peso, los bajos valores de ratio cintura-cadera y una envergadura mayor que la altura. Sin embargo, existe una mayor variedad respecto al resto de índices estudiados, dándose un mayor número de casos de tronco medio para el índice córmico, miembros inferiores largos para el índice de Manuovrier, tronco trapezoidal e intermedio para el índice acromio-iliaco y antebrazo medio y corto para el índice braquial.

AGRADECIMIENTOS: Los autores agradecen su colaboración a la Real Federación Española de Piragüismo, así como a los entrenadores y deportistas que participaron en el estudio.

ALACID, F.; MUYOR, J. M.; ALVERO-CRUZ, J. R.; ISORNA, M. \& LÓPEZ-MIÑNRRO, P. A. Anthropometric indexes in young elite male sprint canoeists. Int. J. Morphol., 30(2):579-582, 2012.

SUMMARY: The objective of this study was to describe the characteristics of the anthropometric indexes in male young sprint canoeists. Body mass, stature, sitting height, arm spam, waist and hip girths, arm and forearm lengths and biacromial and biiliocristal breaths were measured in the 33 canoeists. Anthropometric indexes were obtained: body mass index, waist-hip ratio, relative arm spam and cormic, Manouvrier's, acromial-iliac and brachial indexes. Most paddlers had a healthy weight for height, a low waist-hip ratio, and a higher arm span than stretch stature. Results were more varied in other indexes, with a higher number of paddlers who had intermediate trunk length for Cormic index, long lower limbs for Manouvrier index, intermediate an trapezoidal trunk shape for acromial-iliac index and a medium and short forearm length for brachial index.

KEY WORDS: Anthropometry; Anthropometric indexes; Canoeing. 


\section{REFERENCIAS BIBLIOGRÁFICAS}

Ackland, T. R.; Ong, K. B.; Kerr, D. A. \& Ridge, B. Morphological characteristics of Olympic sprint canoe and kayak paddlers. J. Sci. Med. Sport, 6(3):285-94, 2003.

Aitken, D. A. \& Jenkins, D. G. Anthropometric-based selection and sprint kayak training in children. J. Sports Sci., 16(6):539-43, 1998.

Alacid, F.; López-Miñarro, P. A.; Martínez, I. \& Ferrer-López, V. Anthropometric indexes in young paddlers. Rev. Int. Med. Cienc. Ac., 10(41):58-76, 2011a.

Alacid, F.; Marfell-Jones, M.; López-Miñarro, P. A.; Martínez, I. \& Muyor, J. M. Morphological characteristics of young elite paddlers. J. Hum. Kinet., 27:95-110, 2011b.

Alacid, F.; Muyor, J. M. \& López-Miñarro, P. A. Perfil antropométrico del canoísta joven de aguas tranquilas. Int. J. Morphol., 29(3):83540, 2011c.

Arlettaz, A.; Rieth, N. \& Courteix, D. Assessment of lean body mass and bone mass in specific sites in elite kayak paddlers. Sci. Sports, 19:199201, 2004.

Carrasco, L.; Martínez, E. \& Nadal, C. Perfil antropométrico, somatotipo y composición corporal de jóvenes piragüistas. Rev. Int. Med. Cienc. Ac., 5(19):270-82, 2005.

Carrascosa, A.; Fernández, J. M.; Fernández, C.; Ferrández, A.; López-Siguero, J. P.; Sánchez, E.; Sobradillo, B. \& Yeste, D. Spanish cross-sectional growth study 2008. Part II. Height, weight and body mass index values from birth to adulthood. An. Pediatr. (Barc), 68(6):552-69, 2008.

Cermak, J.; Kuta, I. \& Parizkova, J. Some predispositions for top performance in speed canoeing and their changes during the whole year training program. J. Sports Med. Phys. Fitness, 15(3):243-51, 1975.

Cuesta, G.; Polo, J. M. \& Padilla, S. Correlación entre la marca deportiva obtenida en test de campo y parámetros fisiológicos obtenidos en laboratorio, en piragüistas adolescentes. Apunts, 18:130-42. 1991.

Fry, R. W. \& Morton, A. R. Physiological and kinanthropometric attributes of elite flatwater kayakists. Med. Sci. Sports Exerc., 23(11):12971301,1991

Humphries, B.; Abt, G. A.; Stanton, R. \& Sly, N. Kinanthropometric and physiological characteristics of outrigger canoe paddlers. J. Sports Sci., 18(6):395-9, 2000

López-Miñarro, P. A. \& Alacid, F. Influence of hamstring muscle extensibility on spinal curvatures in young athletes. Sci. Sport, 25(4):188-93, 2010.

López-Miñarro, P. A.; Alacid, F. \& Rodríguez-García, P. L. Comparison of sagittal spinal curvatures and hamstring muscle extensibility among young elite paddlers and non-athletes. Int. Sportmed. J., 11(2):301-12, 2010a

López-Miñarro, P. A.; Muyor, J. M. \& Alacid, F. Sagittal spinal curvatures and pelvic tilt in elite young kayakers. Med. Sport, 63(4):509-519, $2010 \mathrm{~b}$.
López-Miñarro, P. A.; Muyor, J. M. \& Alacid, F. Sagittal spinal and pelvic postures of highly-trained young canoeists. J. Hum. Kinet., 29:51-9, 2011.

Lutoslawska, G.; Sitkowski, D. \& Krawczyk, B. Plasma uric acid response in elite kayakers to 40-s arm cranking before and after training. Biol. Sport, 11(4):233-40, 1994.

Marfell-Jones, M.; Olds, T.; Stewart, A. \& Carter, L. International standards for anthropometric assessment. Potchefstroom, South Africa, ISAK, 2006.

Mirwald, R. L.; Baxter-Jones, A. D.; Bailey, D. A. \& Beunen, G. P. An assessment of maturity from anthropometric measurements. Med. Sci. Sports Exerc., 34(4):689-94, 2002.

Misigoj-Durakovic, M. \& Heimer, S. Characteristics of the morphological and functional status of kayakers and canoeists. J. Sports Med. Phys. Fitness, 32(1):45-50, 1992.

Muyor, J. M.; Alacid, F. \& López-Miñarro, P. A. Morfología sagital del raquis en palistas jóvenes de alto nivel. Int. J. Morphol., 29(3):1047$53,2011$.

Norton, K.; Olds, T.; Olive, S. \& Craig, N. Anthropometry and Sports Performance. En K. Norton \& T. Olds (Eds.), Anthropometrica (pp. 287-364), Sydney, University of New South Wales Press, 1996.

Pacheco, J. L. La proporcionalidad corporal. En F. Esparza (Ed.), Manual de cineantropometría (pp. 95-112). Pamplona: FEMEDE, 1993.

Ridge, B.; Broad, E.; Kerr, D. \& Ackland, T. Morphological characteristics of Olympic slalom canoe and kayak paddlers. Eur. J. Sport Sci., 7(2):107-13, 2007.

Salas-Salvado, J.; Rubio, M. A.; Barbany, M. \& Moreno, B. SEEDO 2007 Consensus for the evaluation of overweight and obesity and the establishment of therapeutic intervention criteria. Med. Clin. (Barc), 128(5):184-96, 2007.

van Someren, K. A. \& Palmer, G. S. Prediction of 200-m sprint kayaking performance. Can. J. Appl. Physiol., 28(4):505-17, 2003.

WHO. Obesity: preventing and managing the global epidemic. Report of a WHO Consultation, Ginebra, World Health Organization, 2000.

Wisniewska, A.; Wojczuk, J.; Obuchowicz-Fidelus, B. \& Lukaszewska, $\mathrm{J}$. Hormones in the four and forty minutes exercise on kayak ergometer. Biol. Sport, 2(2):101-10, 1985.

Dirección para correspondencia:

Fernando Alacid Cárceles

Facultad de Ciencias del Deporte, Universidad de Murcia

C/Argentina, s/n.

30720 Santiago de la Ribera, Murcia

ESPAÑA

Email: fernando.alacid@um.es

Recibido : 18-07-2011

Aceptado: 10-01-2012 